\title{
Bursts, eclipses, dips and a refined position for the luminous low-mass X-ray binary in the globular cluster Terzan 6
}

\author{
J. J. M. in 't Zand ${ }^{1,2}$, F. Hulleman ${ }^{2}$, C. B. Markwardt ${ }^{3,4}$, M. Méndez ${ }^{1}$, E. Kuulkers ${ }^{5}$, R. Cornelisse ${ }^{1,2, \star}$, \\ J. Heise ${ }^{1,2}$, T. E. Strohmayer ${ }^{3}$, and F. Verbunt ${ }^{2}$ \\ 1 SRON National Institute for Space Research, Sorbonnelaan 2, 3584 CA Utrecht, The Netherlands \\ 2 Astronomical Institute, Utrecht University, PO Box 80000, 3508 TA Utrecht, The Netherlands \\ 3 NASA Goddard Space Flight Center, Code 662, Greenbelt, MD 20771, USA \\ 4 Dept. of Astronomy, University of Maryland, College Park, MD 20742, USA \\ 5 ESA-ESTEC, Science Operations \& Data Systems Division, SCI-SDG, Keplerlaan 1, 2201 AZ Noordwijk, The Netherlands
}

Received 21 February 2003 / Accepted 6 May 2003

\begin{abstract}
GRS 1747-312 is a bright transient X-ray source in the globular cluster Terzan 6 with quasi-periodic outbursts approximately every 4.5 months. We carried out 2-60 keV target-of-opportunity observations during eight outbursts with the Proportional Counter Array on the RXTE satellite, for a total exposure time of $301 \mathrm{ks}$, and detect the first unambiguous thermonuclear X-ray bursts from this source. This identifies the compact accretor in this binary as a neutron star. The neutron star identification implies that twelve out of thirteen luminous (above $10^{36} \mathrm{erg} \mathrm{s}^{-1}$ ) X-ray sources in Galactic globular clusters harbor neutron stars, with AC211's nature (in M15) remaining elusive. We observed 24 transitions of eclipses of the X-ray emitting region by the companion star and are able to improve the accuracy of the orbital period by a factor of $10^{4}$. The period is $P=0.514980303(7) \mathrm{d}$. We do not detect a period derivative with an upper limit of $|\dot{P} / P|=3 \times 10^{-8} \mathrm{yr}^{-1}$. Archival Chandra data were analyzed to further refine the X-ray position, and the cluster's center of gravity was re-determined from optical data resulting in a correction amounting to 2 core radii. We find that GRS 1747-312 is $0.2 \pm 0.2$ core radii from the cluster center.
\end{abstract}

Key words. accretion, accretion disks - globular clusters: individual: Terzan 6 - X-rays: binaries - X-rays: bursts X-rays: individual: GRS 1747-312

\section{Introduction}

GRS 1747-312 is a transient X-ray source that was first detected in 1990 with ROSAT (Predehl et al. 1991) and Granat (Pavlinsky et al. 1994). Within the uncertainty of 1 ' it was found to coincide with the globular cluster (GC) Terzan 6. Later this was confirmed by a more accurate ROSAT localization (Verbunt et al. 1995). Terzan 6 is a core-collapsed metal-rich GC which is offset from the Galactic center by 2.6 . The core and half-light radii are 3.' 3 and 26" (Trager et al. 1995), the distance $9.5_{-2.5}^{+3.3} \mathrm{kpc}$ (Barbuy et al. 1997; Kuulkers et al. 2003). It is heavily reddened with $E(B-V)=2.24$ (Barbuy et al. 1997) which is among the highest values of GCs harboring luminous $\left(>10^{36} \mathrm{erg} \mathrm{s}^{-1}\right) \mathrm{X}$-ray sources. There are thirteen luminous X-ray sources in twelve globular clusters in the Galaxy. Thus far, eleven of the thirteen bright GC X-ray sources were proven to harbor neutron stars accreting matter from a Roche-lobe filling low-mass companion star. The proof was

Send offprint requests to: J. J. M. in 't Zand,

e-mail: jeanz@sron.nl

* Present address: University of Southampton, Department of Physics and Astronomy, Southampton, UK. delivered by the detection of type-I X-ray bursts. Such bursts are characterized by fast rises (less than a few seconds), slower exponential-like decays with e-folding decay times between a few seconds and a few tens of minutes, and blackbody spectra that exhibit cooling temperatures during the decay (e.g., Lewin et al. 1993; Strohmayer \& Bildsten 2003). Type-I X-ray bursts are explained as thermonuclear flashes on the surfaces of neutron stars (Maraschi \& Cavaliere 1977; Woosley \& Taam 1976). GRS 1747-312 and AC211 in M15 (White \& Angelini 2001) are the remaining cases for which the nature of the compact objects needs to be resolved. Knowledge about the nature is of interest for studies about the evolution of GCs (i.e., the probabilities of tidal capture and escape probabilities of the black holes versus the lighter neutron stars), particularly now that there is evidence that GCs in other galaxies do contain black hole X-ray binaries (e.g., Angelini et al. 2001; Sarazin et al. 2001).

A previous study of mostly moderately sensitive X-ray data showed that the outbursts of GRS 1747-312 follow each other relatively quickly: In 't Zand et al. (2000) detected five outbursts between 1996 and 1999 with recurrence times of about half a year. It was also established that GRS 1747-312 is one 
of now six known low-mass X-ray binaries that exhibit complete X-ray eclipses; the others are EXO 0748-676 (Parmar et al. 1986; Wolff et al. 2002), MXB 1658-298 (Cominsky \& Wood 1984; Oosterbroek et al. 2001), Her X-1 (Tananbaum et al. 1972), XTE J1710-281 (Markwardt et al. 1999) and AX J1745.6-2901 (Maeda et al. 1996; Kennea \& Skinner 1996). The measured orbital period for GRS 1747-312 is $12.360 \pm 0.009 \mathrm{hr}$, the eclipse duration $43 \mathrm{~min}$.

We initiated a program of dedicated and sensitive targetof-opportunity (ToO) X-ray observations with RXTE to search for signatures regarding the nature of the accretor in GRS 1747-312, in particular type-I X-ray bursts which can unambiguously be identified as thermonuclear flashes in a neutron star atmosphere (like for the other eleven previously mentioned GC cases), and to measure more eclipses to improve the knowledge about the orbital period. The present paper concerns the full analysis of all X-ray bursts, the timing study of the eclipses, and a discussion of the recurrence behavior of the transient outbursts. Furthermore, we analyzed archival Chandra data to determine the most accurate $\mathrm{X}$-ray position thus far. In connection to this we re-determined the cluster's center of gravity from optical data and find that the X-ray source is within one core radius from the center.

\section{ASM and PCA long-term light curves}

The All-Sky Monitor (ASM) on RXTE monitors GRS 1747-312 for about ten months every year, for up to a few tens of times per day during $90 \mathrm{~s}$ dwells. The sensitivity is typically a few tens of $\mathrm{mCrabs}^{1}$ per day of observations (Levine et al. 1996). Although limited, the sensitivity is sufficient to pick up outbursts by GRS 1747-312 which typically peak at $30 \mathrm{mCrab}$. The resulting light curve is presented in Fig. 1. Sixteen outbursts are readily recognized by eye, a few (maybe two) were probably missed at times when the sun passed by GRS 1747-312 and observations were impossible.

Since February 1999 the Proportional Counter Array (PCA, see next section) on RXTE is employed in slow semi-weekly scans of a $16^{\circ} \times 16^{\circ}$ field centered on the Galactic center (Swank $\&$ Markwardt 2001). Fluxes of all point sources in this field are monitored, including that of GRS 1747-312. The observation frequency is one to two orders of magnitude less than that of the ASM observations, but this is compensated by a considerable gain in sensitivity. Figure 2 shows the resulting light curve.

If we assume that two outbursts were missed in the ASM data (between outbursts VIII and IX and between XII and XIII), the average recurrence time is 136 days. However, the recurrence time varies between 76 days (between outbursts VII and VIII, see Fig. 1) and 171 days (between outbursts $\mathrm{XI}$ and XII). There is a clustering of recurrence times in the 130-142 day range: 7 out of 13 securely determined cases are in this range.

Nearly all outbursts have similar shapes and peak intensities of about $380 \mathrm{c} \mathrm{s}^{-1}$ per 5 PCUs or $2 \mathrm{ASM} \mathrm{c} \mathrm{s}^{-1}$, corresponding to 0.03 Crab units; only two outburst are significantly

\footnotetext{
$11 \mathrm{Crab}$ unit is equivalent to $2 \times 10^{-8} \mathrm{erg} \mathrm{cm}^{-2} \mathrm{~s}^{-1}(2-10 \mathrm{keV})$, for a power-law spectrum with photon index 2.1 .
}

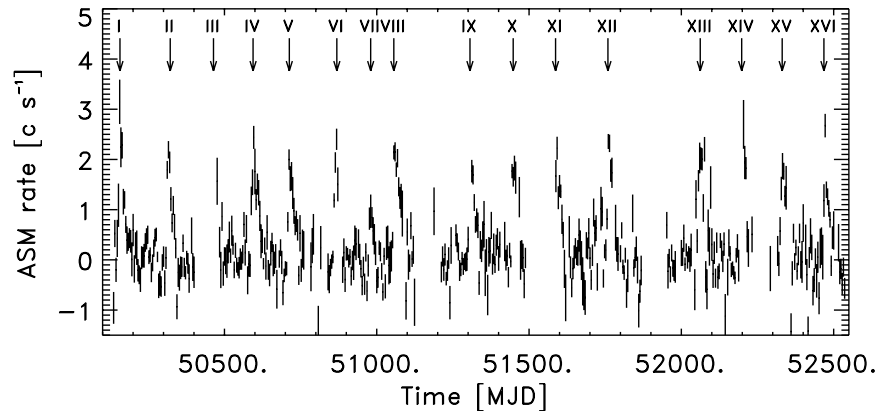

Fig. 1. Time history of GRS 1747-312 photon rate in the ASM, between 6 January 1996 and 26 September 2002. A bias level of $0.5 \mathrm{c} \mathrm{s}^{-1}$ was subtracted. Sixteen outbursts can be recognized. Gaps correspond to times when the sun crosses the field of view of the source and observations are impossible. The time resolution is $4 \mathrm{~d}$.

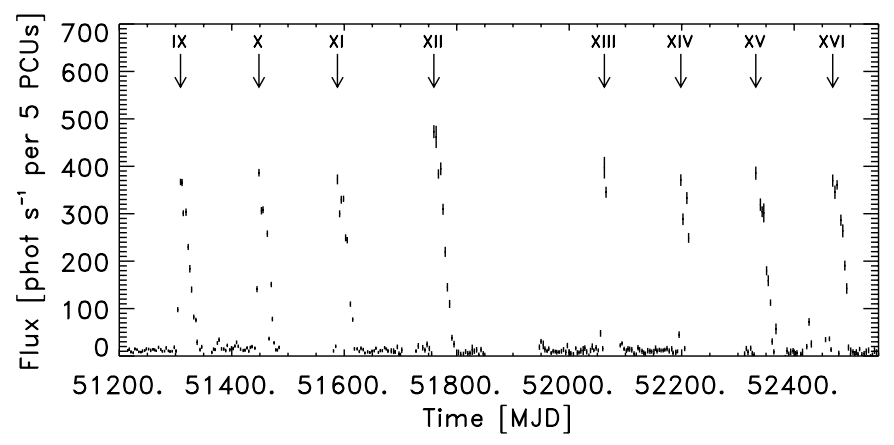

Fig. 2. Time history of GRS 1747-312 photon rate during PCA bulge scans (Swank \& Markwardt 2001; Markwardt \& Swank 2002), between 5 February 1999 and 29 September 2002. Gaps in this plot are also caused by sun passages.

different: the peak intensity of outburst VII is half the usual value and outburst XII is $25 \%$ larger. These deviations are accompanied with extremes in the recurrence time. Figure 3 shows the PCA scan light curve folded with respect to the times of maximum of the seven outbursts. All PCA-detected outbursts last 4 weeks except for the last outburst which had low-level activity preceeding the maximum for one week.

The average intensity from outburst to outburst may accurately be determined from those outbursts that were well sampled by the PCA bulge scans and were accompanied by unambiguous measurements of the recurrence time since the previous outburst. Five outbursts meet these criteria: X, XI, XII, XV and XVI (including the deviating outburst XII). We determined the photon fluence per outburst in a model-independent manner by 1) subtracting a background level as determined from 3 points preceding the peak by at least 7 days; 2) multiplying each data point with the interval time to the next data point; and 3) summing all values. The resulting fluences divided by the recurrence time provides average intensities between outbursts ranging from $50 \pm 2$ to $58 \pm 1 \mathrm{c} \mathrm{s}^{-1}$ per 5 PCUs. The fractional rms value over the 5 measurements is $5 \%$.

\section{RXTE-PCA pointed observations}

The Proportional Counter Array (PCA; for a detailed description, see Jahoda et al. 1996) on RXTE consists of an array of 


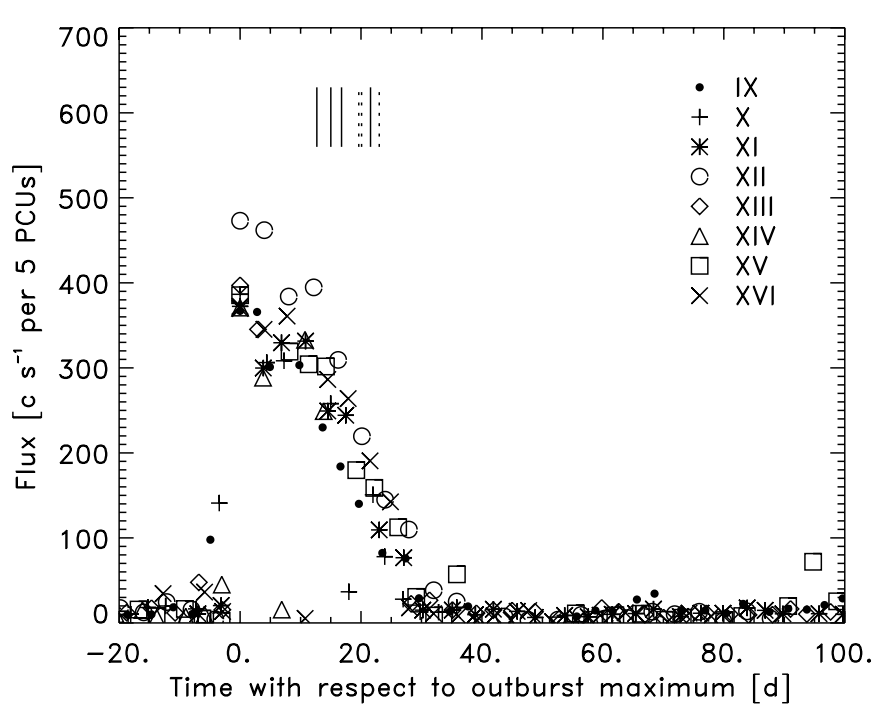

Fig. 3. Same as Fig. 2, except that the times of the 8 maxima have been subtracted (different symbols for different outbursts). The vertical lines indicate the times of detections of type-I X-ray bursts with the PCA (solid) and of burst-like features with the WFCs (dashed). The three low points during the outburst phase have been verified to be caused by eclipses.

5 co-aligned Proportional Counter Units (PCUs) that are sensitive to photons of energy 2 to $60 \mathrm{keV}$ with a total collecting area of $6500 \mathrm{~cm}^{2}$. The spectral resolution is $18 \%$ full-width at half maximum (FWHM) at $6 \mathrm{keV}$ and the field of view is $1^{\circ}$ FWHM. During any observation the number of active PCUs may vary between 1 and 5 .

The data on GRS 1747-312 were obtained in 80 ToO observations that cover eight outbursts in 1998-2002, see Table 1. The total exposure time is $301 \mathrm{ks}$. Thirteen observations (OBSIds 50034-01-05 through 50034-01-06) were scheduled to search for an anticipated outburst, because the usual trigger observations by the ASM and PCA bulge scans were absent due to small angular distances to the Sun. Unfortunately, that outburst did not materialize. On four outbursts wide coverage was obtained (Feb. 2000, Jul. 2000, June 2001 and October 2001). Coverage of the other four outbursts was limited to one or two observations of eclipses. Many of the individual exposures were scheduled to cover either an eclipse ingress or egress and not the full eclipse. An eclipse is rather long and spending much of the exposure budget on that would have diminished the chances to detect an X-ray burst.

\section{X-ray bursts}

\subsection{Detections}

In outbursts XIII and XIV we were succesful in catching bursts. They were detected on June 17 (burst no. 1), October 29 (no. 2), November 2 (no. 3) and 6, 2001 (no. 4); all occurred during the decay phase of an outburst (see Fig. 3). While the bursts all have durations of 10 to $15 \mathrm{~s}$, they rather vary in profile and peak flux (see top panels of Fig. 4) and there is a slight concern that other sources in the field of view are responsible. Therefore,
Table 1. Summary of pointed RXTE observations. The dividing lines indicate different outbursts.

\begin{tabular}{|c|c|c|c|c|}
\hline $\begin{array}{l}\text { Out- } \\
\text { burst }\end{array}$ & ObsID & Dates & $\begin{array}{l}\text { No. } \\
\text { Obs. }\end{array}$ & $\begin{array}{r}\text { Exp. } \\
(\mathrm{s})\end{array}$ \\
\hline VIII & $30427-01-01$ & 1998-09-13 & 1 & 3283 \\
\hline IX & 40419-01-01 & 1999-05-12 & 1 & 3356 \\
\hline \multirow[t]{3}{*}{$\mathrm{X}$} & 40419-01-02 & 1999-09-27 & 2 & 6592 \\
\hline & 40419-01-03 & 1999-10-13 & 1 & 3584 \\
\hline & 40419-01-04 & $1999-10-20$ & 1 & 2432 \\
\hline \multirow[t]{4}{*}{ XI } & 50034-01-01 & $2000-02-18,20,21,24$ & 7 & 26546 \\
\hline & 50034-01-02 & $2000-02-25,26,27,28$ & 9 & 34272 \\
\hline & 50034-01-03 & $2000-03-11,12$ & 2 & 2908 \\
\hline & 50034-01-04 & $2000-03-15$ & 1 & 3518 \\
\hline \multirow[t]{4}{*}{-} & 50034-01-05 & $2000-06-30$ & 7 & 11886 \\
\hline & & $2000-07-1,2,3,4,5,6$ & & \\
\hline & 50034-01-06 & 2000-07-7,8,9,10,11 & 6 & 7791 \\
\hline & & 2000-07-12 & & \\
\hline \multirow[t]{3}{*}{ XII } & 50034-01-07 & $2000-08-10$ & 2 & 13521 \\
\hline & 50034-01-08 & $2000-08-11,12,15$ & 3 & 5717 \\
\hline & 50034-01-09 & 2000-09-09,11 & 3 & $6000^{\dagger}$ \\
\hline \multirow[t]{5}{*}{ XIII } & 60038-01-01 & $2001-05-31$ & 1 & 13860 \\
\hline & $60038-01-03$ & 2001-06-03,06 & 2 & 5609 \\
\hline & 60038-01-04 & $2001-06-10,12,13,16$ & 4 & 20942 \\
\hline & 60038-01-05 & $2001-06-17$ & 2 & 9300 \\
\hline & 60038-01-06 & $2001-07-1,2,5$ & 3 & 6556 \\
\hline \multirow[t]{5}{*}{ XIV } & 60038-01-02 & $2001-10-21,23,24$ & 8 & 43983 \\
\hline & 60038-01-07 & $2001-10-26,27,29,30$ & 8 & 48682 \\
\hline & & $2001-10-31$ & & \\
\hline & 60038-01-08 & $2001-11-02,6,8$ & 3 & 14307 \\
\hline & 60038-01-09 & 2001-11-09 & 1 & 6182 \\
\hline$\overline{X V}$ & 60038-01-10 & 2002-03-14 & 1 & 1377 \\
\hline XVI & 60038-01-11 & $2002-07-16$ & 1 & 1101 \\
\hline
\end{tabular}

$\dagger$ These observations had bad attitude data and were disregarded.

we applied a localization procedure to those bursts that employs the slight offsets that exist between PCUs (Strohmayer et al. 1997). The result is consistent, at the $90 \%$ confidence level, with all bursts coming from within 0.5 of the position of GRS 1747-312. No other bursters are known within this region, although SAX J1752.3-3138 (Cocchi et al. 2001) is at the edge. Furthermore, no otherwise (transiently) bright X-ray sources are known in this region. Given that the bursts all occurred when GRS 1747-312 was in an active state, we conclude that they are from the same source.

Between mid 1996 and mid 2002 the Wide Field Cameras (WFCs; Jager et al. 1997) on BeppoSAX (Boella et al. 1997) were used to monitor a $40^{\circ} \times 40^{\circ}$ field at $5^{\prime}$ resolution around the galactic center during Feb.-Apr. and Aug.-Oct. of each year, with exposure times of $100 \mathrm{ksec}$ to $1 \mathrm{Msec}$ and observation frequencies of 4 to 67 per visibility window (e.g., In 't Zand 2001). The total net exposure time is $7 \mathrm{Msec}$. The bandpass of the 

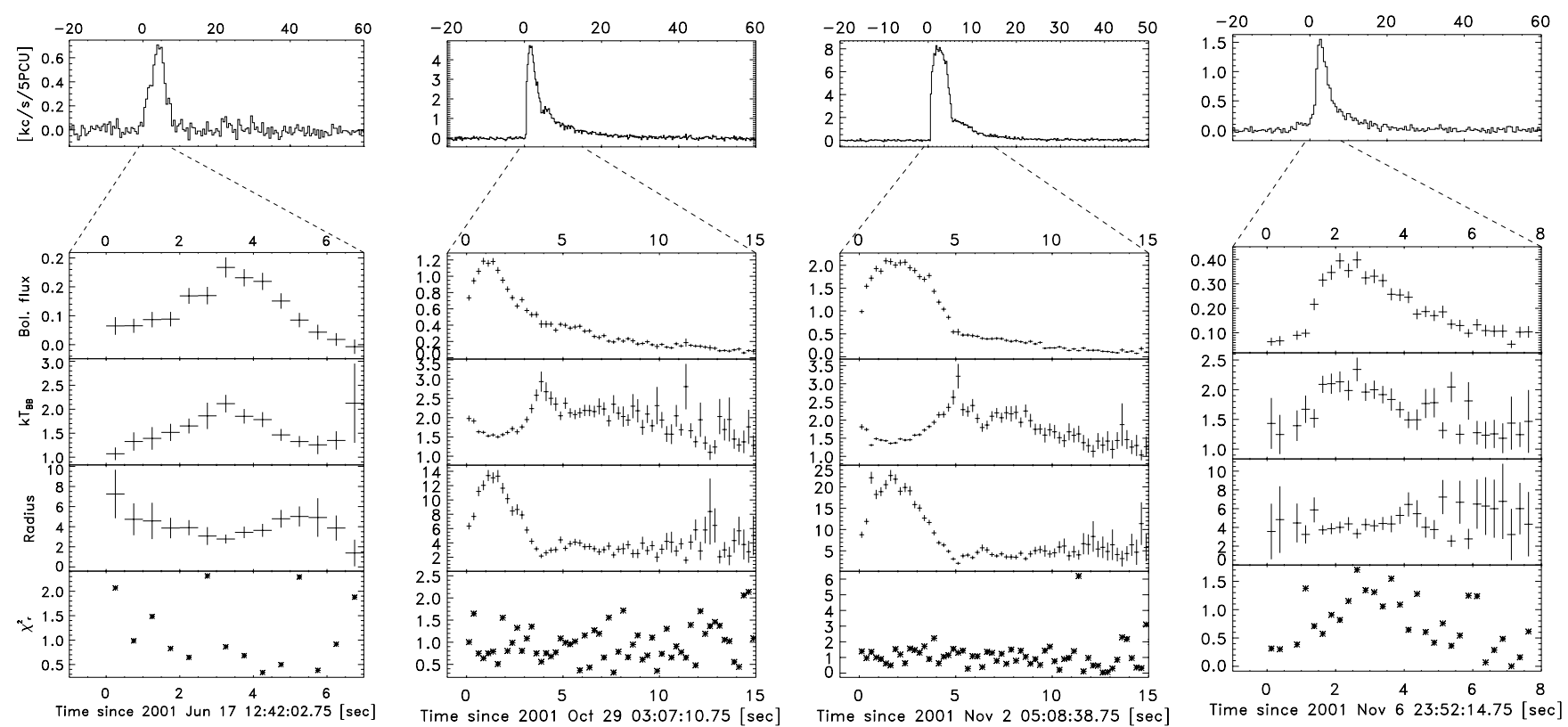

Fig. 4. Time-resolved spectroscopy of the bursts. The upper panel of each burst shows the raw full-bandpass background-subtracted (including the persistent emission from GRS 1747-312) photon count rate, the 2nd panel from above the bolometric absorption-corrected flux in units of $10^{-8} \mathrm{erg} \mathrm{s}^{-1} \mathrm{~cm}^{-2}$, the 3rd panel the blackbody temperature in units of $\mathrm{keV}$, the 4th panel the radius of the spherical blackbody emission area in $\mathrm{km}$ (for a distance of $9.5 \mathrm{kpc}$ ), and the bottom panel the reduced $\chi^{2}$ values for the spectral fit to each time-resolved spectrum. We note that the orbital phases with respect to mid-eclipse for these bursts are $0.86,0.28,0.21$ and 0.49 (see Sect. 5). The abscissa reference times are in UTC.

WFC is 2 to $28 \mathrm{keV}$, and the typical sensitivity is $10 \mathrm{mCrab}$ in one observation. Part of these data were the basis of the detection of the $12.4 \mathrm{hr}$ orbital period of GRS 1747-312 (In 't Zand et al. 2000). The WFC observations revealed the detection of three burst-like features, with durations of less than $10 \mathrm{~s}$ and peak fluxes comparable to those of the brightest PCAdetected bursts. The bursts occurred during the decays of outbursts XI and XII (see Figs. 2 and 3); two were within half a day from each other. Although the data are of insufficient statistical quality to allow a detailed spectral analysis, they do confirm with high localization precision the bursting nature of GRS 1747-312.

\subsection{Time-resolved spectroscopy}

We carried out a time-resolved spectral analysis of the four PCA-detected bursts. Preliminary results were presented in Kuulkers et al. (2003). Event mode data with a resolution of $2^{-11} \mathrm{~s}$ and 64 energy channels were binned to 0.25 through $4 \mathrm{~s}$, the background was subtracted as well as the pre-burst persistent emission from GRS 1747-312, a correction for deadtime was applied, and the spectral data were, between 2 and $20 \mathrm{keV}$, fitted with a single-temperature blackbody model and constant absorption $\left(N_{\mathrm{H}}=1.4 \times 10^{22} \mathrm{~cm}^{-2}\right.$, see In 't Zand et al. 2000). Furthermore, spectral channels were combined so that each spectral bin contained at least 15 photons, to ensure Gaussian statistics and the applicability of the $\chi^{2}$ statistic. The smallest number of spectral bins was 3 . The results are summarized in Fig. 4, together with the raw photon count rate profiles. The spectral fits are generally acceptable. All bursts show a temperature profile that decreases during the decaying phase. The blackbody spectrum and the cooling during decay uniquely identify the bursts as type-I X-ray bursts which are explained as due to thermonuclear flashes in the upper layers of neutron stars (see Sect. 1).

Two of the bursts show a time profile for the emission area which is not constant and anti-correlated with temperature variations. This is indicative of photospheric radius expansion (by a factor of 3 for burst 2 and 6 for burst 3 ) due to near-Eddington fluxes during these events. Remarkably, the unabsorbed bolometric peak flux during these two bursts differs by a factor of 1.8 .

\subsection{Search for burst oscillations}

We searched the four X-ray bursts for oscillations. Light curves were produced at the full time resolution of the data, $2^{-11} \mathrm{~s}$, starting $\sim 5 \mathrm{~s}$ before the onset of the bursts and extending up to times when the intensity had decayed back to within $\sim 10 \%$ of the preburst value. We used data for the whole energy band, nominally 2-60 keV, and from all the detectors that were on during each burst (three detectors during burst 1, 3, and 4; and four detectors during burst 2). We calculated one Fourier power spectrum of the whole burst for each burst, and power spectra of 1,2, and 4-s intervals covering the whole duration of the bursts. In the case of the 1,2, and 4-s power spectra, to increase the sensitivity to transient oscillations lasting less than the time length of the power spectra, we set the beginning of the interval for which we calculated the power spectrum $0.125,0.250$, and $0.5 \mathrm{~s}$, respectively, after the beginning of the previous interval (the power spectra were therefore not independent). For the brightest burst (no. 3), we also calculated power spectra of 1) 0.25 and $0.5 \mathrm{~s}$ intervals, shifting by $1 / 32$ and $1 / 16 \mathrm{~s}$ at a time; and 2) for $>6 \mathrm{keV}$ photons only. 
We did not find any significant oscillation in the $0.025-1024 \mathrm{~Hz}$ frequency range in any of the bursts. Because of the rapid change in intensity during the bursts, we calculated upper limits separately for intervals during which the source intensity was larger than $90 \%$ of the peak intensity, and the rest of the data. For the faintest burst (no. 1) the observed count rate was a factor of $\sim 7$ lower than for the brightest burst (no. 3), and therefore the upper limits vary considerably from burst to burst. The $95 \%$ confidence upper limits for oscillations (for the method, see Vaughan et al. 1994) in bursts 1 and 4 during the bright and weak parts are $29.8 \% \mathrm{rms}$ and $63.8 \% \mathrm{rms}$, respectively. For bursts 2 and 3, the $95 \%$ confidence upper limits in the bright and weak parts are $11.5 \% \mathrm{rms}$ and $47.3 \% \mathrm{rms}$, respectively.

In the 11 sources that currently are known to exhibit burst oscillations, the oscillations may reach high amplitudes $(>50 \%$ rms) during short intervals (typically $0.1 \mathrm{~s}$ ) if they occur during the rising phase and low amplitudes $(<15 \%)$ during the decaying phase (for a recent review, see Strohmayer \& Bildsten 2003). Therefore, our upper limits are not very constraining.

\section{Eclipses and dips}

Twelve eclipse ingresses and twelve egresses were observed, in twenty-one eclipses distributed over seven outbursts. Two eclipses were observed completely; for another one the ingress and egress was covered with a data gap during the eclipse; for the rest only the ingress or egress was covered. Timing of the transitions provides the means to improve the accuracy of the orbital ephemeris significantly. The orbital period thus far had only a $31 \mathrm{~s}$ accuracy (In 't Zand et al. 2000). More accuracy enables studies of changes in the orbital period. To increase the time baseline, we include the timing of the egress observed with the BeppoSAX Narrow Field Instruments on 1998 September 6 (In 't Zand et al. 2000). Thus, the time baseline is $3.9 \mathrm{yr}$.

Ingress and egress last between 30 and $40 \mathrm{~s}$. We took the mid points between minimum and maximum flux as a reference for the timing of the eclipse transitions. The transition profiles appear too variable to trust methods that involve profile fitting. Therefore, we resorted to the following procedure. As a baseline, we used standard 1 data which provides $0.125 \mathrm{~s}$ resolution. The times were corrected to the solar system barycenter ${ }^{2}$. We identified the eclipses in a light curve of the whole data set at a resolution of $1 \mathrm{~s}$ and selected data stretches of $200 \mathrm{~s}$ centered on the initial midpoint determinations (by eye) of ingress or egress. The light curves of the center $120 \mathrm{~s}$ parts of these data stretches are displayed in Fig. 5. One ingress had to be excluded from the analysis (at MJD 52060) because the data stream stopped before total eclipse was reached; one egress was excluded because the source was too faint (at MJD 51601). We determined the weighted average of the initial and final $50 \mathrm{~s}$ of each data stretch, except for the very last egress in which a small flare-like feature needed to be avoided (see Fig. 5). This excludes the eclipse transitions. The time when the flux reaches the halfway point between these two averages is determined by

\footnotetext{
${ }^{2}$ using the faxbary routine in ftools version 5.2.
}

Table 2. Times and durations of all eclipse transitions observed thus far with sufficient sensitivity

\begin{tabular}{|c|c|c|c|c|}
\hline $\begin{array}{l}\text { Time } \\
\text { MJD (TDB) }\end{array}$ & $\begin{array}{l}\text { error } \\
\text { (s) }\end{array}$ & $\overline{n^{\dagger}}$ & $\begin{array}{r}\text { deviation } \\
\text { (s) }\end{array}$ & $\begin{array}{r}\text { duration* } \\
\text { (s) }\end{array}$ \\
\hline \multicolumn{5}{|c|}{ ingresses } \\
\hline 51448.798094 & 0.40 & -1199 & $+1.2(+3.0 \sigma)$ & $27.9 \pm 1.9$ \\
\hline 51599.687282 & 2.18 & -906 & $-2.5(-1.2)$ & $56.7 \pm 14.8$ \\
\hline 51601.747211 & 1.43 & -902 & $-1.9(-1.3)$ & $34.1 \pm 6.9$ \\
\hline 51614.106731 & 2.61 & -878 & $-2.5(-1.0)$ & $22.7 \pm 6.8$ \\
\hline 51767.055894 & 0.36 & -581 & $-1.6(-4.5)$ & $30.8 \pm 2.1$ \\
\hline 52063.684558 & 0.50 & -5 & $-1.2(-2.2)$ & $33.6 \pm 3.4$ \\
\hline 52066.259477 & 0.47 & 0 & $+0.3(+0.7)$ & $29.7 \pm 2.6$ \\
\hline 52077.074080 & 0.45 & 21 & $+1.8(+3.9)$ & $25.5 \pm 2.3$ \\
\hline 52077.589014 & 1.42 & 22 & $-2.2(-1.6)$ & $31.3 \pm 5.2$ \\
\hline 52203.244242 & 0.36 & 266 & $+0.5(+1.5)$ & $25.9 \pm 1.8$ \\
\hline 52347.438718 & 0.47 & 546 & $-0.4(-0.9)$ & $21.6 \pm 2.0$ \\
\hline \multicolumn{5}{|c|}{ egresses } \\
\hline $51062.592982^{x}$ & 3 & -1949 & $+3.6(+1.2 \sigma)$ & \\
\hline 51594.567535 & 0.52 & -916 & $-2.6(-5.0)$ & $31.6 \pm 3.2$ \\
\hline 51600.747403 & 1.14 & -904 & $+6.4(+5.7)$ & $39.3 \pm 8.3$ \\
\hline 51602.807303 & 4.08 & -900 & $+4.6(+1.1)$ & $68.1 \pm 22.1$ \\
\hline 51615.166779 & 4.68 & -876 & $+0.2(+0.1)$ & $50.5 \pm 10.0$ \\
\hline 51768.115903 & 0.50 & -579 & $-1.3(-2.6)$ & $36.1 \pm 3.6$ \\
\hline 52060.624742 & 0.60 & -11 & $+2.4(+3.9)$ & $36.2 \pm 4.1$ \\
\hline 52066.289531 & 0.95 & 0 & $+2.9(+3.1)$ & $46.9 \pm 6.5$ \\
\hline 52073.499250 & 0.97 & 14 & $+2.5(+2.5)$ & $41.5 \pm 5.9$ \\
\hline 52077.619037 & 1.81 & 22 & $-2.3(-1.3)$ & $44.7 \pm 10.4$ \\
\hline 52206.364193 & 0.81 & 272 & $+5.2(+6.4)$ & $42.2 \pm 5.9$ \\
\hline 52471.578956 & 0.40 & 787 & $-1.6(-4.1)$ & $28.1 \pm 2.1$ \\
\hline
\end{tabular}

${ }^{\dagger}$ Number of orbits.

$¥$ Deviation with respect to linear fit.

${ }^{*}$ See text for definition.

${ }^{\times}$BeppoSAX-NFI observation.

identifying the data point closest to this halfway flux, selecting the 17 data points nearest to this point, making a least-squares linear fit to these 17 data points, and finally calculating the interpolated time of the halfway point. This procedure was developed more or less empirically. It avoids complications due to changing transition profiles, particularly near the start and end of each transition. There may be a bias introduced by the variability of the source outside the eclipse but we estimate this to be negligible compared to the statistical accuracy. The statistical errors are of order $1 \mathrm{~s}(1 \sigma)$ in this analysis. The results of the timing are given in Table 2 . In addition to the timing of the mid-transition points, we determined a measure for the transition duration by calculating the time it takes the linear fit to bridge the weight averages of the initial and final $50 \mathrm{~s}$ stretches.

From these data we determine the ephemeris for the ingresses and egresses. The deviations from these solutions (the "O-C" values) are presented in Table 2 and Fig. 6. Ten out of the twenty values are larger (in a positive or negative sense) than $3 \sigma$, but there are no clear trends in $\mathrm{O}-\mathrm{C}$ and we conclude that there is no evidence for systematic changes in the orbital period. Rather we suspect that the larger-than-statistical errors are due to imperfections of the method. Assuming this is the case, we multiply the errors of the linear solutions of 

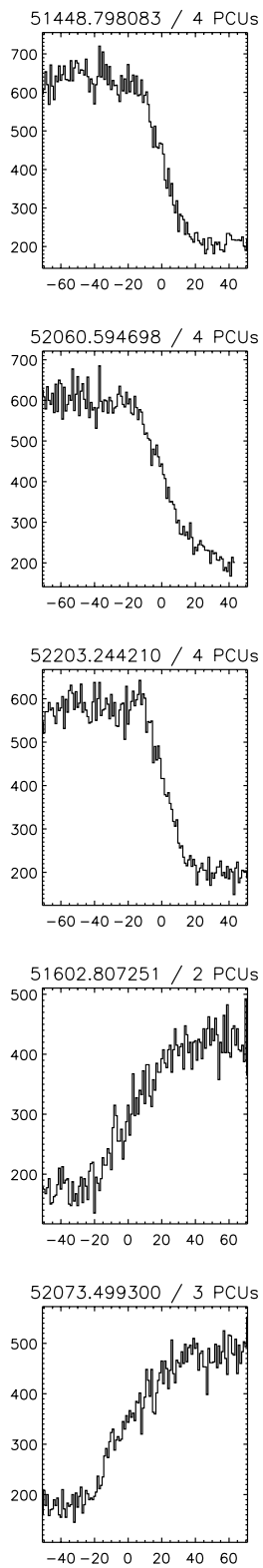
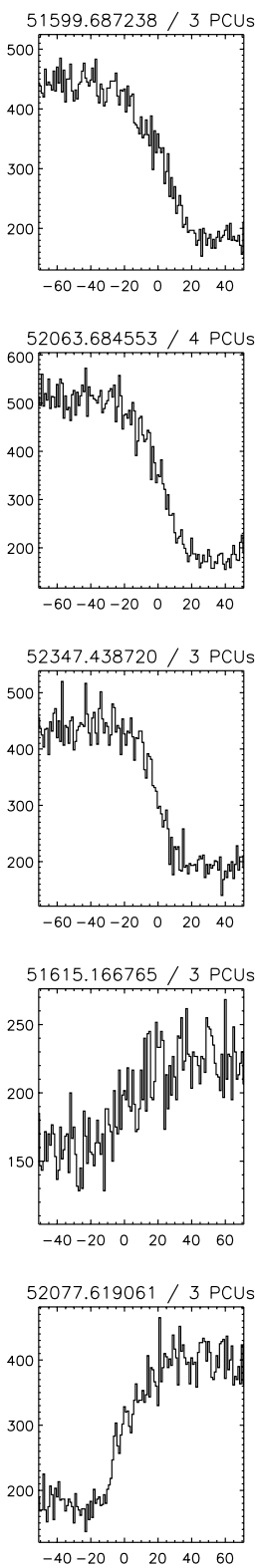
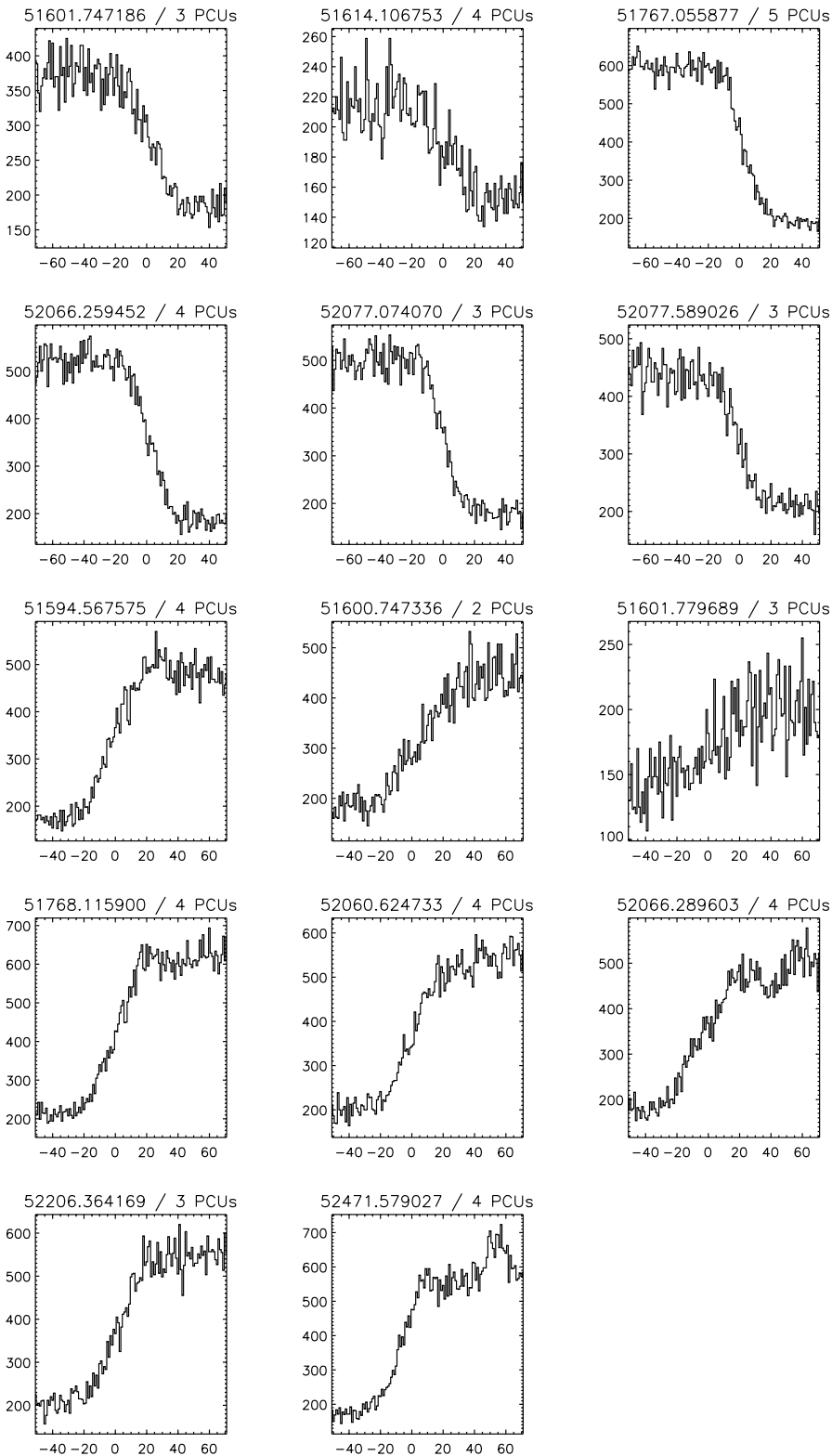

Fig. 5. Light curves of $120 \mathrm{~s}$ data stretches around 24 eclipse transitions in GRS 1747-312. The abscissa refers to time in second, with a reference time that is indicated above each plot in MJD (using Terrestrial Time and corrected to solar system barycenter; TDB), and the ordinate shows the photon rate normalized for 5 PCUs (note that the actual number of active PCUs is not constant over all 24 data stretches and is indicated at the top of each plot).

the ingresses and egresses by the square-root of the $\chi_{\text {red }}^{2}$ values ( $\sqrt{6.6}$ for the ingresses and $\sqrt{15.7}$ for the egresses), thereby effectively forcing $\chi_{\text {red }}^{2}$ to 1 . The ephemeris for the ingresses is $T_{\mathrm{i}}(n)=\operatorname{MJD}(\mathrm{TDB}) 52066.259473(5)+0.514980311(8) n$ and for the egresses $T_{\mathrm{e}}(n)=\mathrm{MJD}$ (TDB) 52066.289497(10)+ $0.514980275(15) n$. The difference in the two derived orbital periods is $3.1 \mathrm{~ms}$ with an error of $1.7 \mathrm{~ms}$. We take for the orbital period the weighted mean of $0.514980303(7) \mathrm{d}$. This is only $0.15 \mathrm{~s}$ shorter than the previous determination (In 't Zand et al. 2000). We determine an upper limit to the period derivative of $|\dot{P} / P|=1 \times 10^{-16} \mathrm{~s}^{-1}$ or $3 \times 10^{-8} \mathrm{yr}^{-1}$. The average eclipse duration is $2596(2) \mathrm{s}$ (or $43.27 \mathrm{~min}$, or 0.05834 orbital periods).
It may be that the larger-than-statistical errors in the midtransition times are due to intrinsic properties rather than imperfections of the method. A similar phenomenon has been observed in EXO 0748-676. In addition to a long term period evolution trend, a significant amount of jitter is detected in that source (Wolff et al. 2002). Wolff et al. demonstrate that the eclipse phasing of EXO 0748-676 is compatible with a random walk process. They speculate that the companion star may become significantly tidally distorted, enough so that the times of eclipse ingress and egress can be shifted from their nominal value. The long term period evolution may be driven by spinorbit coupling via the quadrupole moment of the companion star. In the present analysis we are only interested in the 


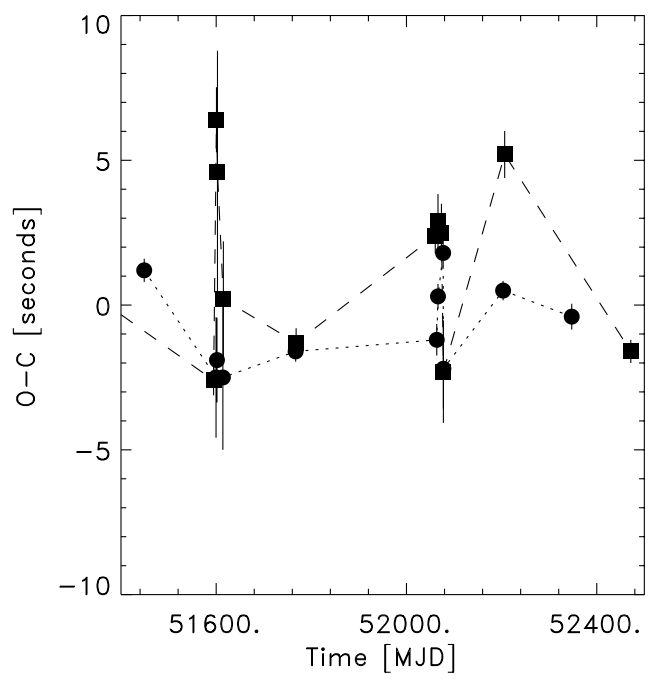

Fig. 6. Observed minus calculated ("O-C") diagram for the eclipse ingresses (circular symbols connected by a dotted line) and egresses (rectangular symbols connected by a dashed line) as observed with RXTE, with respect to the respective ephemerises (see text).

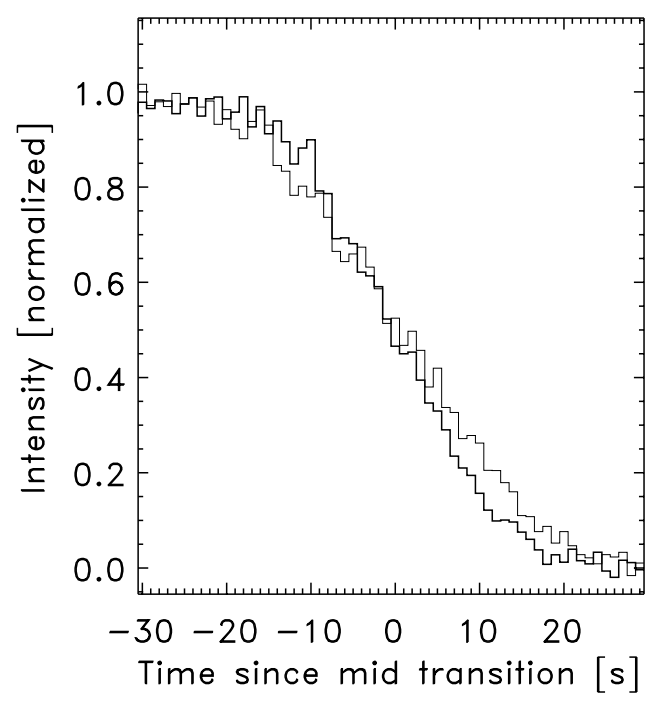

Fig. 7. Average ingress (thick) and egress profile (thin) after renormalization of both and mirroring the time axis of the egress profile. The average profiles have been constructed by aligning individual profiles at the time of mid transition (see Table 2).

long-term orbital period evolution of GRS 1747-312 and we regard the jittering as noise.

The average transition profiles are given in Fig. 7. Surprisingly, there is a difference between the average ingress and egress. In particular, the average egress appears to progress about 1.3 times slower than the ingress. This is confirmed by the averages of the durations as given per transition in Table 2: they are $27.3 \pm 0.8 \mathrm{~s}$ for ingress and $33.8 \pm 1.3 \mathrm{~s}$ for egress.

In Fig. 8 we present the average ingress and egress profiles for two separate bands: $2-5 \mathrm{keV}$ and $5-20 \mathrm{keV}$. These show that the eclipse lasts approximately $25 \mathrm{~s}$ longer in the low-energy band, indicating absorption in the atmosphere of the obscuring companion star. This is spectrally confirmed by the BeppoSAX measurement (In 't Zand et al. 2000).
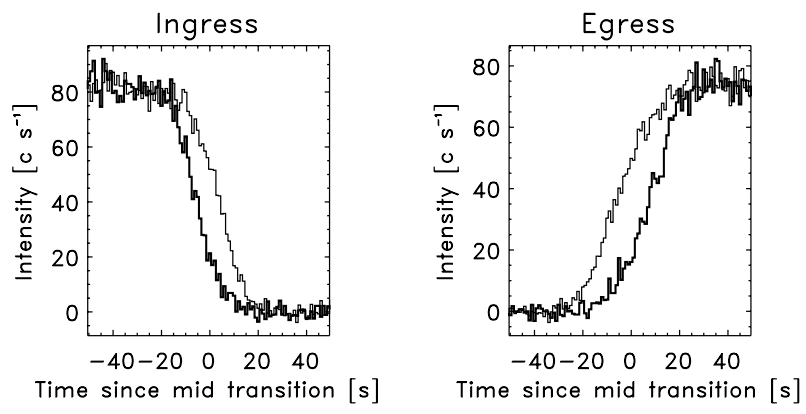

Fig. 8. In thick lines, the average ingress profile (left) and egress profile (right) in the $2-5 \mathrm{keV}$ band; in thin lines in the $5-20 \mathrm{keV}$ band. The averages have been taken over 6 good-quality ingresses and 8 egresses. Background subtraction consisted of two steps: first for particle-induced and cosmic background (using the pcabackest tool in ftools version 5.2) and second for residual emission during total eclipse. The 5-20 keV intensities have been renormalized to match the plateau values of the $2-5 \mathrm{keV}$ curves.

We tested the transition profiles in the two bandpasses with a simple model involving an isothermal spherical atmosphere in hydrostatic equilibrium where the density is an exponential function of height, assuming a constant source spectrum that is equal to that measured with BeppoSAX (In 't Zand et al. 2000). This model turns out to give a good representation of the data. Both the low and high-energy profiles are well reproduced as is the lag between them. We find neutron star traverse times over the scale height of $6.0 \pm 0.5$ and $8.5 \pm 0.5 \mathrm{~s}$ for the average ingress and egress respectively, with goodness-of-fits of $\chi_{\text {red }}^{2}=$ 0.893 (400 d.o.f.) and 0.989 (386 d.o.f.) respectively. With the eclipse duration this results in upper limits for the true scale height in terms of stellar radius of 0.2 to $0.3 \%$. We also tested linearly extended sources instead of a point source, as would be expected for a nearly edge-on accretion disk, and find fits that are only marginally better by about $3 \%$ in $\chi_{\text {red }}^{2}$, with sourcesize equivalent traverse times of 14 and $20 \mathrm{~s}$ and scale heights only 0.5 to $1.0 \mathrm{~s}$ smaller than for a point source. We regard the evidence for an extended source as weak.

As in other eclipsing low-mass X-ray binaries (LMXBs; EXO 0748-676, MXB 1658-298, XTE J1710-281), GRS 1747-312 shows dipping activity (for an example, see Fig. 9). Dips are thought to be caused by coverage of the emission region by irregular thickening of the accretion disk (White \& Swank 1982). Thanks to the large inclination angle, inferable from the occurrence of eclipses, the presence of dips is not unexpected. Dipping activity was observed during outbursts XI (during two different binary orbits), XII (one orbit) and XIV (one orbit). The orbital phase with respect to mid-eclipse was between 0.58 and 0.74 . The dip depth never exceeds $70 \%$, indicating that the emission region is never completely obscured by the disk irregularities. The dips last typically a fraction of a minute.

\section{Accurate positions of X-ray source and cluster center}

Chandra observed GRS 1747-312 on March 9, 2000, from 5:44:44 to $8: 41: 16$ UT (PI J. Grindlay). This observation was 


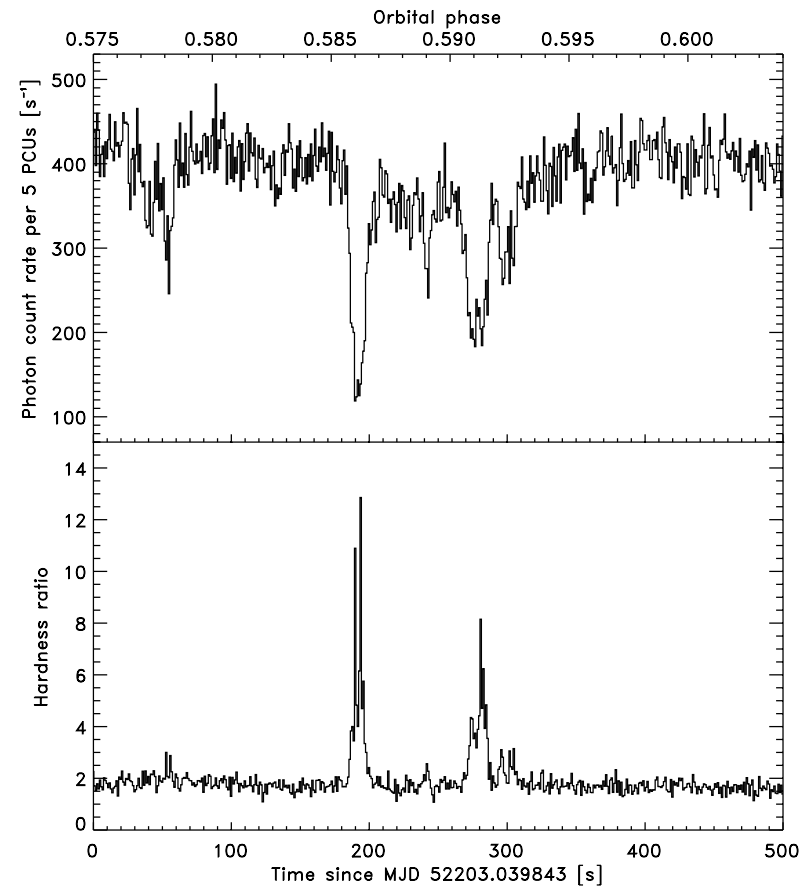

Fig. 9. Upper panel: 2-20 keV light curve during a period of dipping activity; lower panel: $5-10$ to $2-5 \mathrm{keV}$ hardness ratio. The times are solar-system barycentered and the photon rates have been background subtracted. The background count rate includes particle-induced background, diffuse background and the local Galactic background as determined from the full eclipse observed on MJD 52206 at which time the same PCUs were employed.

triggered by our RXTE observations showing the source to be active. The High Resolution Camera HRC-I was used for a total net exposure time of $9964 \mathrm{~s}$. No grating was used. The HRC-I is a microchannel plate detector with a field of view of $30^{\prime} \times 30^{\prime}$ and an angular resolution of 0.' 4 (FWHM), a bandpass of 0.1 to $10.0 \mathrm{keV}$ with $227 \mathrm{~cm}^{2}$ effective area at $1 \mathrm{keV}$, and minimal spectral resolution (Murray et al. 2000). We extracted the data from the public archive for an analysis of the position. Such an analysis was performed previously by Revnitsev et al. (2002). However, we anticipated to improve on that by attempting an astrometric solution of the image through X-ray detections of cataloged stars.

GRS 1747-312 was significantly detected. A total of $15 \times 10^{3}$ photons were detected and the intensity was $1.50 \pm$ $0.01 \mathrm{~s}^{-1}$. Slow variability was observed with a $\approx 20 \%$ modulation around the average on a time scale of $10^{3} \mathrm{~s}$. Applying the 'celldetect' tool from CIAO (version 2.2) to the image, we find four more point sources with a net number of photons in excess of 10, and three sources between 6 and 10 . We determine the pixel positions of all eight sources. Using the satellite aspect solution and requiring a match within $1^{\prime \prime}$ (the absolute astrometric accuracy is quoted by the Chandra team as $0 . ' 6$ to $0 . ' 8$ at $90 \%$ confidence), we tentatively identify three X-ray detections of stars in the USNO-B1.0 catalog (Monet et al. 2003). They are USNO0587-0575246 $\left(b_{\text {USNO }}=\right.$ $\left.12.46, r_{\mathrm{USNO}}=11.45\right), \mathrm{USNO} 0587-0575103$ (14.79 and 13.32) and USNO0586-0575381 (16.10 and 14.19). From the same optical image we determined that the probability for chance coincidence of a star brighter than $r_{\mathrm{USNO}}=14.19$ with one $1^{\prime \prime}$-radius circle is $7 \times 10^{-4}$ and we conclude that the identifications are correct. We determined (X-ray minus optical) offset corrections of $0 \prime^{\prime \prime} 68 \pm 0 .{ }^{\prime} 12$ and $0{ }^{\prime} 53 \pm 0 . ' 12$ in $\alpha_{2000.0}$ and $\delta_{2000.0}$ respectively. Applying these corrections, we arrive at a position for GRS 1747-312 of $\alpha_{2000.0}=17^{\mathrm{h}} 50^{\mathrm{m}} 46^{\prime \prime} .862$, $\delta_{2000.0}=-31^{\circ} 16^{\prime} 28^{\prime \prime} .86$ with an error radius of $0 . \prime 4(95 \%$ confidence). This position is consistent with the position determined by Revnitsev et al. (2002), but has an 1.8 times better accuracy (when compared at the same confidence level).

On 17 May 1994, optical images of Terzan 6 were acquired with the $3.55 \mathrm{~m}$ New Technology Telescope (NTT) of the European Southern Observatory, using the SUSI-1 camera which employed a $1024 \times 1024 \mathrm{CCD}$ at the Nasmyth focus B, giving a plate scale of 0.' 13 per pixel (Barbuy et al. 1997). These images were taken under excellent seeing conditions ( $\left.0{ }^{\prime \prime} 6\right)$ in $I$ (300 s), $V(480 \mathrm{~s})$ and Gunn $z$ (120 s) filters. We carried out astrometry of the $V$ and $z$ plates relative to the USNO-B1.0 catalog. Centroids of observed star profiles were determined by Gaussian fitting. The astrometric solution consisted of a zero point position, plate scale, and position angle on the sky. 14 catalog stars were identified in the $V$ image with deviations less than $0{ }^{\prime} 4$, and 19 stars in the $z$ image with deviations less than $0 . ' 6$. The rms values of the measured star positions with respect to the cataloged positions is $0 .{ }^{\prime \prime} 20$ in right ascension and 0. ' 14 in declination $(0,25$ and 0.24 respectively for $z$ ). Both solutions are less than 0.' 15 apart.

The position of the center of Terzan 6 that is used throughout the literature $\left(\alpha_{2000.0}=17^{\mathrm{h}} 50^{\mathrm{m}} 46^{\prime \prime} 44, \delta_{2000.0}=\right.$ $-31^{\circ} 16^{\prime} 31^{\prime \prime}$. 4 ) is due to the original discovery plate (Terzan 1968, see also Terzan 1971). From comparison with Fig. 10 it is clear that this position is substantially off the densest concentration. Therefore, we re-determined the center through the iterative centroiding method introduced by Auriere (1982; see also Picard \& Johnston 1994). In this method the (unweighted) average position is taken over all resolved stars within a certain radius from a trial position. This average becomes the trial position in the next iteration. The procedure is repeated until the average converges. For Terzan 6, a radius of 15 " was chosen on the basis of the cluster's appearance in our image. The resulting center is $\alpha_{2000.0}=17^{\mathrm{h}} 50^{\mathrm{m}} 46^{\prime \prime} .854, \delta_{2000.0}=-31^{\mathrm{o}} 16^{\prime} 29.38^{\prime \prime}{ }^{\prime} 4$ with an uncertainty of $0 .{ }^{\prime \prime} 6$ as evaluated by testing different values for the radius. The ultimate determination of the center is based on the average position of 219 stars. This newly determined center is 5.'7 away from the old position. We note that this correction does not affect the structural parameters of the cluster because those were determined completely from relative positions (Trager et al. 1995).

The Chandra position of GRS $1747-312$ is $0 . \prime 5$ from our determination of the center which is equivalent to 0.2 core radii. This confirms the suggestion from the sub-day orbital period and the moderately high collision number (Verbunt 2001) that this binary was formed due to tidal capture or exchange encounter.

No sources belonging to Terzan 6 other than GRS 1747-312 were unambiguously detected in the Chandra image. Outside the point spread function of GRS 1747-312, the upper limit for the intrinsic luminosity for a $0.3 \mathrm{keV}$ black body spectrum with 


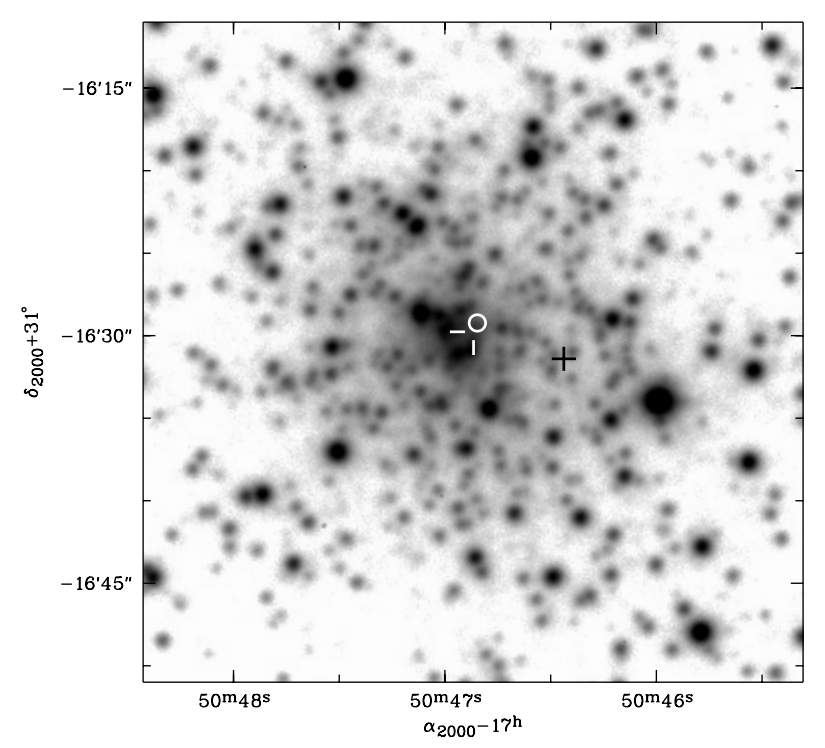

Fig. 10. Gunn $z$ plate of Terzan 6 (Barbuy et al. 1997). The weakest stars are approximately $z=23$. The position of the bright transient is marked by a white circle (with error radius of 0.'5), the newly determined position of the center of gravity is indicated by two white lines, and the old position by a black cross.

$N_{\mathrm{H}}=1.4 \times 10^{22} \mathrm{~cm}^{-2}$ is approximately $1.3 \times 10^{33} \mathrm{erg} \mathrm{s}^{-1}$. The point-spread function is roughly given by a Gaussian profile with a standard deviation of 0 .' 4 . It degrades the sensitivity to faint sources by a factor of more than two for distances closer to GRS $1747-312$ than 1 ". 8 .

\section{Discussion}

\subsection{Outburst behavior}

The $2-10 \mathrm{keV}$ peak flux is mostly about $7 \times 10^{-10} \mathrm{erg} \mathrm{cm}^{-2} \mathrm{~s}^{-1}$. Broad-band measurements during outburst VIII allowed an extrapolation to $0.1-200 \mathrm{keV}$ of $1.0 \times 10^{-9} \mathrm{erg} \mathrm{cm}^{-2} \mathrm{~s}^{-1}$ (In 't Zand et al. 2000). The outburst duration is always 4 weeks. The fractional rms in the average intensity from outburst to outburst is 5\%. If we assume that the spectrum does not change drastically from outburst to outburst, the luminosity averaged over all times (outburst as well as quiescence) is $1.5 \times 10^{36} \mathrm{erg} \mathrm{s}^{-1}$ and the equivalent mass accretion rate, assuming $100 \%$ efficiency of transforming the liberated gravitational energy to radiation, is about $1.2 \times 10^{-10} M_{\odot} \mathrm{yr}^{-1}$ (i.e., $1 \%$ of Eddington). One accurately measured outburst (XII) was delayed by $25 \%$ of the average wait time. The peak of that outburst was also $25 \%$ larger while the duration was the same, so that the average luminosity remained constant. It must be a rather constant mass transfer rate from the companion star to the accretion disk which is responsible for the regular outburst behavior. A similar behavior is observed in the Rapid Burster in the GC Liller 1: it shows a quasi-periodic outbursts whose period of about 210 days suddenly switched to 100 days in Nov. 1999 in combination with a factor-of-two decrease in the peak flux (Masetti 2002). Another well-documented case is Aql X-1, with at least 31 outbursts detected thus far (e.g., Šimon 2002), but its recurrence time is not so well defined and there does not seem to be as strong a correspondence between recurrence time and fluence per outburst.

The transient nature that many LMXBs exhibit is generally thought to be related to thermal-viscous instabilities in the accretion disk where the thermal conditions are predominantly determined by heating through X-rays from the central object (e.g., Smak 1983; Osaki 1996; van Paradijs 1996; Lasota 2001, Šimon 2002). These instabilities become important and the source becomes transient if the average X-ray luminosity is below a critical value (van Paradijs 1996, see also White et al. 1984). The onset of an outburst occurs if the surface column density at any location in the accretion disk exceeds a critical value. As a consequence the temperature rises, the viscosity increases, and heating fronts travel in and outward through the disk. Depending on the radius of the ignition, differently sized outbursts may result (outward fronts are expected to be less efficient, possibly resulting in smaller outbursts if the ignition occurs in the inner parts of the disk). Furthermore, the ignited parts of the disk may irradiate other cool parts of the disk and ignite those. The increased viscosity results in a depletion of the accretion disk which may be complete or partial. The latter is more likely to happen if the ignition radius is small. The recurrence time, peak flux and outburst duration depend on quite a number of parameters and effects, but it seems obvious that the quasi-periodicity at least testifies to a rather stable mass transfer rate from the secondary. The similar recurrence times of GRS 1747-312, Aql X-1 and the Rapid Burster probably reflect similar orbital periods (19 hrs for Aql X-1, unknown for the Rapid Burster), similar mass transfer rates and the fact that they are all neutron star systems without considerable truncation of the disks that may result in decades-long recurrence times such as in black hole systems (e.g., Dubus et al. 2001).

\subsection{Eclipse timing and evolutionary constraints}

As illustrated by Horne et al. (1985), the eclipse duration expressed as a fraction of the orbital period constrains the mass ratio and inclination. For the low-mass X-ray binary in Terzan 6 we find that the inclination must be higher than 74.5 , and the mass of the mass donor must be higher than 0.07 times the mass of the neutron star. Thus, for a canonical $1.4 M_{\odot}$ neutron star, the lower limit on the mass of the secondary is $0.1 M_{\odot}$; a likely upper limit is $0.8 M_{\odot}$. Kepler's third law yields a semi-major axis of 3.0 to $3.6 R_{\odot}$ for these masses. This implies an average orbital velocity of the secondary with respect to the primary between 296 and $356 \mathrm{~km} \mathrm{~s}^{-1}$.

The eclipse timing resolves an orbital period that has remained constant within $|\dot{P} / P|<3 \times 10^{-8} \mathrm{yr}^{-1}$ for almost 4 years. This corresponds to a lower limit to the time scale of any orbital period change of $3 \times 10^{7} \mathrm{yr}$ which is mildly constraining. Only one LMXB has evidence of a shorter time scale: $P / \dot{P}=2 \times 10^{7} \mathrm{yr}$ in EXO 0748-676 (Wolff et al. 2002).

We may compare these observational constraints with theory as follows. In a globular cluster, the most massive star still on the main sequence has a mass of about $0.8 M_{\odot}$. A mainsequence star of this mass, or lower, cannot fill its Roche lobe in an orbit of $0.515 \mathrm{~d}$. Thus, the donor of GRS 1747-312 must 
be larger than a main-sequence star of the same mass. If it is larger due to stellar evolution into a (sub)giant, then its initial mass was the turnoff mass of $0.8 M_{\odot}$. For a neutron-star mass of $1.4 M_{\odot}$ and a companion of $0.8 M_{\odot}$, the orbital period gives a distance between the two stars of $3.6 R_{\odot}$, and a Roche lobe for the donor of $1.2 R_{\odot}$. At such a radius, a subgiant expands on a time scale longer than $R / \dot{R} \simeq 10^{10} \mathrm{yr}$. If the donor has already reduced its mass by mass transfer to the neutron star, its Roche lobe will be somewhat smaller (e.g. $0.9 R_{\odot}$ at a mass of $0.4 M_{\odot}$ ), and the expansion time scale longer. For conservative mass transfer, in which no mass or angular momentum is lost from the binary, the time scales for the mass transfer and for changes in the orbital period are set by the expansion time scale of the donor, i.e. $M / \dot{M} \sim P / \dot{P} \sim R / \dot{R}$. The time averaged mass transfer rate of $\dot{M} \simeq 1.4 \times 10^{-10} M_{\odot} \mathrm{yr}^{-1}$ derived above, and the limit of the period change $\dot{P} / P<3 \times 10^{-8} \mathrm{yr}^{-1}$ are both compatible with the expansion rate of a subgiant donor star. The low mass transfer rate furthermore indicates that the time scale for angular momentum loss from the binary through magnetic braking is longer than $\sim 10^{10} \mathrm{yr}$.

An alternative explanation for an expanded donor star would be that the donor still contains a fair amount of thermal energy left over from its capture by the neutron star (e.g., Verbunt 1994). Since the life time of such a source would be set by its thermal time scale, this possibility is less probable a priori; and the arguments above show that it is not necessary as all the properties of the low-mass X-ray binary may be explained with an ordinary subgiant donor.

\subsection{Eclipse profile}

The transition profiles in GRS 1747-312 are remarkably stable, when compared to other LMXBs. Ingress and egress durations range from -30 to $+30 \%$ with respect to the average. In EXO 0748-676, for instance, the variation is $100 \%$ (Wolff et al. 2002). The stability may be due to either a relative stability of magnetic activity on the secondary, or a relative smoothness of the accretion process (and associated X-ray irradiation of the secondary).

We observed a difference in the eclipse duration at $2-5 \mathrm{keV}$ and 5-20 keV energies and modeled this by assuming an isothermal spherical atmosphere in hydrostatic equilibrium where the density is an exponential function of height. This simple model reproduces the eclipse duration difference for a scale height, as projected on the path of the neutron star line of sight through the atmosphere, of $2.0 \times 10^{3} \mathrm{~km}$ for the ingress and $2.8 \times 10^{3} \mathrm{~km}$ for the egress. The model reproduces the curvature on both ends of the transition profiles and the spectral change over the transition. The true scale height depends on the precise inclination angle. If the primary and secondary are 1.4 and $0.8 M_{\odot}$ respectively, the inclination angle would be 74.5 , the secondary radius $1.2 R_{\odot}$ and the true scale height $1.2 \times 10^{3} \mathrm{~km}$. This is about 2 times larger than expected, but this could be explained by X-ray irradiation of the secondary.

The $\approx 25 \%$ difference between the average ingress and egress duration has not been reported before in any other
LMXB eclipser. Perhaps the relative stability of the transitions is what makes this measurement possible in this particular source. The difference is indicative of a similar difference in the atmosphere's scale height between the leading and trailing hemisphere of the secondary. Day et al. (1988) predicted such a scale height difference, in the same sense, from a Coriolis force acting on supersonic flows on the secondary's surface as invoked by X-ray irradiation. This will increase the scale height on the trailing hemisphere while decreasing it on the leading hemisphere. Whether this model is viable to GRS $1747-312$ is hard to verify without the detection of the secondary star (note that the secondaries have been detected in the well-documented eclipsers Her X-1, MXB 1658-298 and EXO 0748-676).

\subsection{Bursts}

The first-time detection of type-I X-ray bursts unambiguously proves that the compact object in GRS 1747-312 is a neutron star. This leaves AC211 in M15 as the sole bright LMXB in a Galactic globular cluster for which no nature has been determined of the compact object. Finding bursts in this object is going to be at least as difficult as for GRS 1747-312, because it also is a high-inclination system. In fact, the central source is obscured by the accretion disk rim all the time (e.g., Ilovaisky et al. 1993) and bursts can only be detected indirectly through scattered X-rays. Another complication for AC211 is that it is accompanied by another bright and bursting LMXB at a distance of only 2.'7 (White \& Angelini 2001) so that only Chandra and XMM-Newton will be able to localize bursts accurately enough.

All seven bursts from GRS 1747-312 are relatively short suggesting that the flashes occur in a hydrogen-poor/heliumrich layer which, according to burst theory (Fujimoto et al. 1981), must have been formed by stable hydrogen fusion. For that to happen, the accretion rate must be higher than about $1 \%$ of Eddington. This value is consistent with the observations. The highest observed peak flux translates to a luminosity of $\left(2.3_{-1.1}^{+1.9}\right) \times 10^{38} \mathrm{erg} \mathrm{s}^{-1}$ for a distance of $9.5_{-2.5}^{+3.3} \mathrm{kpc}$ (Kuulkers et al. 2003). This value is consistent with the Eddington limit as expected for a hydrogen-rich atmosphere. The four bursts that were well measured with the PCA have the following interesting characteristics:

1. there is a large range of peak fluxes from 0.2 to $2.1 \times$ $10^{-8} \mathrm{erg} \mathrm{cm}^{-2} \mathrm{~s}^{-1}$

2. the two radius expansion bursts have fast rise times below $1 \mathrm{~s}$, while the others have slower rise times of at least $2 \mathrm{~s}$. In fact, burst no. 1 has a rise and decay of similar duration which is atypical for a type-I burst;

3. one radius-expansion burst (no. 3) has a 1.8 times higher unabsorbed bolometric peak flux than the other;

4. the minimum apparent radius of a spherical blackbody is as low at $4 \mathrm{~km}$.

We suspect that at least three of these atypical observations are associated with the high inclination angle of the binary. This suspicion is motivated by a recent observation of MXB 1658-298 by Wijnands et al. (2002). They observed two bursts during a period of dipping activity which had peak 
intensities that were four and ten times smaller than of most ordinary bursts and which showed slow rises that were similar to the decay times. We should point out that there is one characteristic which makes the two MXB 1658-298 bursts different from the GRS 1747-312 ones: they were separated by merely (a record) $50 \mathrm{~s}$ which suggests that incomplete burning (e.g., Fujimoto et al. 1987) may have been important in determining some of the burst characteristics. Short recurrence times can be excluded for GRS 1747-312: the data coverage around the two small bursts without additional bursts is at least $1200 \mathrm{~s}$. However, it is our impression that the short recurrence time does not explain the slow rise times. No dipping was observed around the bursts of GRS 1747-312, but we note that the two small bursts were observed at orbital phases of 0.87 (burst 1) and 0.49 (burst 4 ) that are more favorable for obscuration effects than for the bright bursts (the phase is 0.28 for burst 2 and 0.21 for burst 3).

Regarding the factor-of-1.8 peak flux difference between the two radius expansion bursts, this is similar as in another well-measured system. Galloway et al. (2003) were able to gather observations of 61 photospheric radius expansion bursts from $4 \mathrm{U} 1728-34$ (=GX 354-0). They found a variation in the peak flux by a factor of 1.5 which is correlated with spectral variations of the persistent emission. They suggest that a variation in the geometry of the accretion disk may be at work, for instance precession of a warped disk.

Acknowledgements. We are indebted to Evan Smith for his tireless efforts to schedule the many time-constrained TOO observations that led to this paper. JZ and EK acknowledge financial support from the Netherlands Organization for Scientific Research (NWO).

\section{References}

Angelini, L., Loewenstein, M., \& Mushotzky, R. F. 2001, ApJ, 557, L35

Auriere, M. 1982, A\&A, 109, 301

Barbuy, B., Ortolani, S., \& Bica, E. 1997, A\&A, 122, 483

Boella, G., Butler, R. C., Perola, G. C., et al. 1997, A\&AS, 122, 299

Cocchi, M., Bazzano, A., Natalucci, L., et al. 2001, A\&A, 387, L37

Cominsky, L. R., \& Wood, K. S. 1984, ApJ, 283, 765

Day, C. S. R., Tennant, A. F., \& Fabian, A. C. 1988, MNRAS, 231, 69

Dubus, G., Hameury, J.-M., \& Lasota, J.-P. 2001, A\&A, 373, 251

Fujimoto, M. Y., Hanawa, T., \& Miyaji, S. 1981, ApJ, 247, 267

Fujimoto, M. Y., Sztajno, M., Lewin, W. H. G., \& van Paradijs, J. 1987, ApJ, 319, 902

Galloway, D. K., Psaltis, D., Chakrabarty, D., \& Muno, M. P. 2003, ApJ, submitted [astro-ph/0208464]

Horne, K. 1985, MNRAS, 213, 129

Ilovaisky, S. A., Auxière, M., Koch-Miramond, L., Chevalier, C., Cordoni, J.-P., \& Crow, R. A. 1993, A\&A, 270, 139

In 't Zand, J. J. M. 2001, In Proc. 4th INTEGRAL workshop (Sep. 2000, Alicante, Spain), ed. A, Giminez, V. Reglero \& C. Winkler, 463

In 't Zand, J. J. M., Kuulkers, E., Bazzano, A., et al. 2000, A\&A, 355, 145

Jager, R., Mels, W. A., Brinkman, A. C., et al., 1997, A\&AS, 125, 557 Jahoda, K., Swank, J. H., Stark, M. J., et al. 1996, Proc. SPIE, 2808, 59
Kennea, J. A., \& Skinner, G. K. 1996, PASJ, 48, L117

Kundu, A., Maccarone, Th., \& Zepf, S. 2002, ApJ, 574, L5

Kuulkers, E., Hartog, P. R., In 't Zand, J. J. M., et al. 2003, A\&A, 399, 663

Lasota, J.-P. 2001, NewAR, 45, 449

Lewin, W. H. G., van Paradijs, J., \& Taam, R. E. 1993, Space Sci. Rev., 62, 223

Levine, A. M., Bradt, H., Cui, W., et al. 1996, ApJ, 469, L33

Maeda, Y., Koyama, K., Sakano, M., Takeshima, T., \& Yamauchi, S. 1996, PASJ, 48, 417

Maraschi, L., \& Cavaliere, A. 1977, in Highlights in Astron., 4, 127

Markwardt, C. B., Swank, J. \& Marshall, F. E. 1999, IAUC 7120

Markwardt, C. B., \& Swank, J. 2002, presented at the April 2002 meeting of the High-Energy Astrophysics Division meeting in Albuquerque, abstract X11.007

Masetti, N. 2002, A\&A, 381, L45

Monet, D., Levine, S., Canzian, B., et al. 2003, ApJ, in press [astro-ph/0210694]

Murray, S. S., Austin, G., Chappell, J., et al. 2000, Proc. SPIE, 4012, 68

Oosterbroek, Parmar, A.N., Sidoli, L., In 't Zand, J. J. M., \& Heise, J. 2001, A\&A, 376, 532

Osaki, Y. 1996, PASP, 108, 39

Parmar, A. N., White, N. E., Giommi, P. \& Gottwald, M. 1986, ApJ, 308, 199

Pavlinsky, M. N., Grebenev, S. A., \& Sunyaev, R. A. 1994, ApJ, 425, 110

Picard, A., \& Johnston, H. M. 1994, A\&A, 283, 76

Predehl, P., Hasinger, G., \& Verbunt, F. 1991, A\&A, 246, L21

Revnitsev, M. G., Trudolyubov, S. P., \& Borozdin, K. N. 2002, Astron. Lett., 28, 276

Sarazin, C., Irwin, J., \& Bregman, J. 2001, ApJ, 556, 533

Šimon, V. 2002, A\&A, 151, 167

Smak, J. 1983, ApJ, 272, 234

Strohmayer, T. E., Jahoda, K., Giles, A. B., \& Lee, U. 1997, ApJ, 486, 355

Strohmayer, T. E., \& Bildsten, L. 2003, in Compact Stellar X-Ray Sources, ed. W. H. G. Lewin, \& M. van der Klis (Cambridge University Press), in press [astro-ph/0301544]

Swank, J., \& Markwardt, C. 2001, In Proc. New Century of X-ray Astronomy, ed. H. Inoue, \& H. Kunieda, PASP Conf. Ser., 94

Tananbaum, H., Gursky, H., Kellogg, E. M., et al. 1972, ApJ, 174, L143

Terzan, A. 1968, C.r. Acad. Sci. Paris 267, série B, 1245

Terzan, A. 1971, A\&A, 12, 477

Trager, S. C., King, I. R., \& Djorgovski, S. 1995, AJ, 109, 218

Van Paradijs, J., 1996, ApJ, 464, L139

Vaughan, B. A., van der Klis, M., Wood, K. S., et al. 1994, ApJ, 435, 362

Verbunt, F. 1994, A\&A, 285, L21

Verbunt, F. 2001, in Proc. " $\omega$ Cen, a unique window in astrophysics", ed. F. van Leeuwen, G. Piotto, \& J. Hughes, ASP Conf. Ser, 289 [astro-ph/0111441]

Verbunt, F., Bunk, W., Hasinger, G., \& Johnston, H. M. 1995, A\&A, 300,732

White, N. E., \& Swank, J. H. 1982, ApJ, 253, L61

White, N. E., Swank, J. H., \& Kaluzienski, J. L. 1984, in High Energy Transients in Astrophysics, ed. S. Woosley, AIP, p. 31

White, N. E., \& Angelini, L. 2001, ApJ, 561, L101

Wijnands, R., Muno, M. P., Miller, J. M., et al. 2002, ApJ, 566, 1060

Wolff, M. T., Hertz, P., Wood, K. S., Ray, P. S., \& Bandyopadhyay, R. M. 2002, ApJ, 575384

Woosley, S. E., \& Taam, R. E. 1976, Nature, 263, 101 